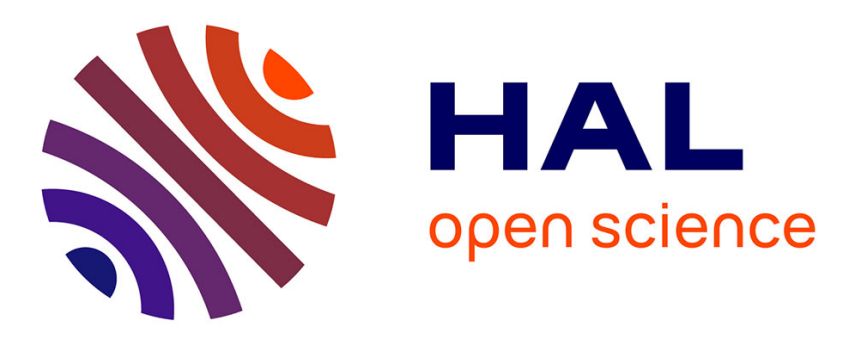

\title{
Low-frequency band gap in cross-like holey phononic crystal strip
}

Shan Jiang, Hongping Hu, Vincent Laude

\section{To cite this version:}

Shan Jiang, Hongping Hu, Vincent Laude. Low-frequency band gap in cross-like holey phononic crystal strip. Journal of Physics D: Applied Physics, 2018, 151 (4), pp.045601 (9). hal-02131465

\section{HAL Id: hal-02131465 \\ https://hal.science/hal-02131465}

Submitted on 16 May 2019

HAL is a multi-disciplinary open access archive for the deposit and dissemination of scientific research documents, whether they are published or not. The documents may come from teaching and research institutions in France or abroad, or from public or private research centers.
L'archive ouverte pluridisciplinaire HAL, est destinée au dépôt et à la diffusion de documents scientifiques de niveau recherche, publiés ou non, émanant des établissements d'enseignement et de recherche français ou étrangers, des laboratoires publics ou privés. 


\title{
Low-frequency band gap in cross-like holey phononic crystal strip
}

\author{
Shan Jiang ${ }^{1,2}$, Hongping $\mathrm{Hu}^{1,2^{*}}$ and Vincent Laude ${ }^{3}$ \\ ${ }^{1}$ Department of Mechanics, Huazhong University of Science and Technology, \\ Wuhan 430074, P. R. China \\ 2 Hubei Key Laboratory of Engineering Structural Analysis and Safety \\ Assessment, Huazhong University of Science and Technology, Wuhan 430074, \\ P. R. China \\ ${ }^{3}$ Franche-Comté Electronique Mécanique Thermique et Optique, CNRS UMR \\ 6174, Université de Bourgogne Franche-Comté, Besançon 25030, France \\ *Corresponding author, E-mail: huhp@hust.edu.cn
}

\begin{abstract}
A silicon-based cross-like holey phononic crystal strip is proposed for the control of elastic waves in the field of Micro-electro-mechanical systems (MEMS). The goal is to obtain a broad bandgap at low frequencies with a lightweight structure. In this respect, the effects of varying the in-plane and the out-of-plane geometry parameters are discussed. After design, a gap-to-midgap ratio of $47 \%$ is obtained with an intermediate filling fraction of the solid material and a small thickness of the strip. The band gap can be moved to an extremely low frequency range while keeping the strip significantly smaller than previously reported phononic crystal strips. The transmission property through a finite number of periods agrees well with the band structure of the infinite system. The proposed phononic crystal strip could for instance be used as an isolating anchor for elastic wave resonators in MEMS.
\end{abstract}

PACS numbers: $43.35 .+\mathrm{d}, 62.30 .+\mathrm{d}$

Keywords: Phononic crystal, phononic crystal strip, Micro-electro-mechanical systems (MEMS), cross-like hole, low-frequency band gap

\section{Introduction}

Phononic crystals (PnCs) have experienced great development during the past decades. The most salient trait of PnCs is the existence of frequency band gaps, within which the propagation of elastic waves is forbidden whatever the polarization and wave vector. They have 
potential application in many fields like vibration attenuation, acoustic isolation, sound collimation, wave filtering, etc. [1]. Previous works have shown that band gaps are determined by several factors, such as the material properties of each component, the geometry parameters, and the lattice symmetry [2]. In general, for a given lattice constant, a wider band gap at lower frequencies means $\mathrm{PnC}$ devices can be made more compact and lightweight. Many studies have been correspondingly conducted to lower and widen band gaps [3-17], which can be boiled down to three different strategies: Bragg scattering inherent to the periodic structure [3-8], local resonances provided by additional material or structure added to the unit cell [9-12], and coupling between both approaches [13-15]. Many novel and creative topologies have been proposed and optimized to obtain broad band gaps $[16,17]$. The study of PnCs has thus become one of the most active and fast-growing disciplines in physics (condensed matter physics, wave propagation in inhomogeneous and periodic media) and engineering (acoustics, ultrasonic, mechanical engineering, electrical engineering) $[18,19]$.

In the field of Micro-electro-mechanical systems (MEMS), devices are very sensitive to low-frequency elastic waves, including energy loss via supports and anchors, and noise contamination from environmental vibration sources. Thus, it is critical for MEMS applications to control elastic waves. In recent years, many works have focused on silicon-based PnCs to control elastic waves propagating through MEMS [20-30]. Yet, researchers still face the challenge that highly integrated MEMS design imposes strict restrictions on area and volume of structures. Establishing topologies that optimize the performance of PnCs and meet application demands of MEMS remains a salient problem.

Compared with the more commonly used two-dimensional PnC plates, one-dimensional $\mathrm{PnC}$ strips can save more space and are compatible with integration, making them prospective candidates for vibration shielding and energy loss reduction in MEMS. By now, research on PnC strips has mainly focused on two kinds of structures: pillared PnC strips and holey PnC strips [24-27, 31]. Pillared PnC strips (and corresponding plates) comparatively received more attention due to their additional property of local resonance [14, 32-36]. However, hard, heavy, and tall pillars are required to obtain wide band gaps basing on strong material contrast with the supporting beam. It was observed that when the ratio of the pillar height to the thickness of the beam is close to 3 , a band gap begins to open, and that when the ratio increases to 10 , the band 
gap reaches its maximal width [32]. These requirements not only bring puzzle regarding size and space, but also result in fabrication and stability challenges.

As for holey PnC strips, band gaps mainly arise from periodic Bragg scattering. Since the geometry parameters are necessarily commensurate with the wavelength, conventional topologies can hardly open low frequency band gaps in micro-PnCs. Even at the sacrifice of size and space, the band gap width remains far from expectations. Up to now, most researches have been limited to convex (such as circular or regular polygonal) holes. Furthermore, for convex-holey structures, a small filling fraction of solid material is usually required to obtain a relative wide band gap. This fact exacerbates fabrication and mechanical stability issues that already exist in MEMS. As an alternative topology, concave holes involve multiple geometry parameters that are useful to tune band gaps. More importantly, the introduction of concave holes can induce a broad band gap, as was shown for both phononic $[4,5]$ and photonic crystals (PtCs) [37]. Nevertheless, concave-holey PnC strips are still lacking research.

In this paper, we propose a cross-like holey phononic crystal strip. The primitive cell has a cross-like hole in the center and a pair of symmetric stubs on the sides. We aim at obtaining low-frequency and wide band gaps with strong attenuation, while at the same time obtaining a lightweight structure. After classifying the modal shapes of the unit cell, we broaden the main band gap by lowering and flattening the relevant bands. A comparison with conventional convex-holey PnC strips is conducted to illustrate the advantages of cross-like holey strips. The influence of geometry parameters on the band gaps is further discussed. A gap-to-midgap ratio of up to $47 \%$ is obtained when the geometry is tailored properly. Finally, in order to evaluate the performance of the PnC for isolating vibrations in MEMS, transmission through a finite structure is examined by using finite element analysis. In summary, the following advantages of the proposed PnC strip are demonstrated: 1) broad band gap, 2) band gap with lower frequency than previously published topologies, 3) small size, and 4) intermediate filling fraction of the solid material, thus avoiding the shortcomings of strips with either small or large filling fraction.

The paper is organized as follows. The PnC strip model and finite element method (FEM) are described in Section 2 where two parameters are defined to describe the properties of the strip. Section 3 then presents band structures and eigenmodes for the infinite periodic strip, and 
transmission spectra for a finite periodic strip. The dependence of the characteristics of the $\mathrm{PnC}$ upon geometry parameters is further discussed. Finally, conclusions are drawn in Section 4.

\section{Phononic crystal strip and calculation method}

The cross-like silicon phononic crystal strip with lattice constant $a$ is depicted in figure 1 . As shown in figure 1 (b), the unit cell consists of a cross-like hole in the center and of four entrant square holes at the corners. It is noted that this topology can also be regarded as four lumps connected by four narrow connectors. The cross-like hole in the center is described by parameters $b$ and $c$, while the side length of the square hole at each corner is $d / 2$. The width of the connectors is thus $w=(a-c-d) / 2$. The thickness of the strip is $h$. All these parameters are normalized against the lattice constant $a$ in the following. The whole strip is made of single crystal silicon, the mass density is $\rho=2320 \mathrm{~kg} / \mathrm{m}^{3}$ and the elastic constants are $c_{11}=165.7$ $\mathrm{GPa}, c_{12}=64.1 \mathrm{GPa}$, and $\boldsymbol{c}_{44}=79.6 \mathrm{GPa}$, respectively. The $y$ and $x$ axes of the strip coincide with the [010] direction of a (100)-oriented silicon wafer.

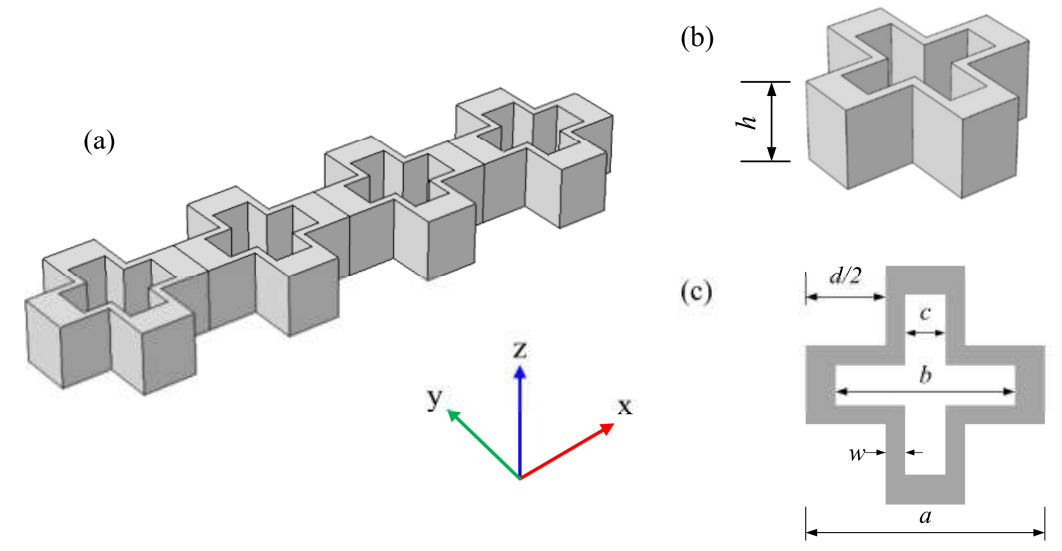

Figure 1. (a) Schematic of the cross-like holey PnC strip with the orientation of (100)-oriented silicon wafer. (b) Primitive unit cell. (c) Plane view of the unit cell with definition of geometry parameters.

Computations are performed by using the finite element method (FEM) with software COMSOL Multiphysics. The model object built in COMSOL is exported and is then imported to MATLAB for the benefit of nested loops. This procedure is used to evaluate the effect of geometry parameters on the band gaps, notably for cases involving multiple geometry parameters. The geometry parameters are normalized by lattice constant $a$. The non-dimensional method is applied in the calculation. 
The governing equation for propagation of elastic waves in anisotropic material can be written as

$$
\frac{\partial}{\partial x_{j}}\left(c_{i j k l} \frac{\partial u_{k}}{\partial x_{l}}\right)=\rho u_{i} \quad(i, j, k, l=1,2,3)
$$

where $x_{j}(j=1,2,3)$ correspond to the $x, y$ and the $z$ axes. $c_{i j k l}$ is the elastic tensor and $u_{i}$ represents the displacement components $u_{x}, u_{y}$ or $u_{z}$. A superimposed dot denotes derivative with respect to time. To compute band structures, Bloch periodic boundary conditions are applied on the two opposite sides of the unit cell along the $x$ direction, and other boundaries are left free. The Bloch-Floquet theorem implies that the displacements have to satisfy the condition

$$
\mathbf{u}(\mathbf{r}+\mathbf{a})=e^{\mathrm{ik} \cdot \mathbf{a}} \mathbf{u}(\mathbf{r})
$$

where $\mathbf{k}$ is the wave vector, $\mathbf{r}$ denotes the position vector and $\mathbf{a}$ is the lattice vector. All eigenmodes can be obtained by sweeping the wave vector $\mathbf{k}$ along the edges of the first irreducible Brillouin zone, which is here one-dimensional. In the band structures, normalized frequencies $\left(\varpi a / 2 \pi c_{t}\right)$ are functions of the reduced wavenumber $(k a / 2 \pi)$, where $c_{t}=4683 \mathrm{~m} / \mathrm{s}$ is the transverse wave velocity along the [110] direction of silicon. The normalized frequencies $\left(\varpi a / 2 \pi c_{t}\right)$ and the non-dimensional band gap depend on the normalized geometry parameters, but are independent on the lattice constant. For this reason, a larger lattice constant can lead to a lower frequency band gap if same normalized geometry parameters are applied. According to the non-dimensional calculation results, one can conveniently determine the lattice constant $a$ to meet the actual requirements on the frequency and the width of the band gap in the practical application.

In order to evaluate the kinetic energetic ratio in a given direction, we define normalized values for the $x, y$, or $z$ polarization components of elastic wave as

$$
e_{i}=\int_{V} u_{i}^{2} \mathrm{~d} V / \int_{V}\left(u_{x}^{2}+u_{y}^{2}+u_{z}^{2}\right) \mathrm{d} V, i=x, y, z
$$

where $u_{i}$ stands for displacement components $u_{x}, u_{y}$ or $u_{z} . V$ denotes the volume of the unit cell. The kinetic energetic ratio can be used to distinguish whether an elastic wave belongs to longitudinal wave or transverse wave or their mixture. As an example, when propagating in $x$-direction, the wave is longitudinal wave if $e_{x}=1$, or it is transverse wave if $e_{y}=1$ or 
$e_{z}=1$

The relative bandwidth $\mathrm{BG} \%$ is defined as a measure on the performance of the phononic crystal and is the ratio of the gap width to the mid gap frequency,

$$
\mathrm{BG} \%=\frac{2\left(f_{\text {top }}-f_{\text {bot }}\right)}{f_{\text {top }}+f_{\text {bot }}} \%
$$

where $f_{\text {top }}$ and $f_{\text {bot }}$ are the bounding frequencies of the band gap. Frequency dependence can be avoided by the non-dimensional parameter. One can consider both the width and frequency position of a band gap at the same time. A higher BG\% means a wider gap, or a lower mid gap frequency, or both. Thus as for the phononic crystal, the higher BG\% indicates the better performance.

\section{Results and discussion}

Figures 2(a)-2(c) present the band structures of the proposed PnC strip. The geometry parameters are set as $b / a=0.625, c / a=0.25, d / a=0.325$ and $h / a=0.2$. The kinetic energetic ratios $e_{i}$ along the three spatial axes are plotted in colour for every band. We can distinguish every band from the colour of the kinetic energetic ratios along the three spatial axes. A band gap range from 0.082 to 0.132 is visible, corresponding to a $\mathrm{BG} \%$ of $47 \%$. It is well known that the uniform slab has three bands starting from $\omega=0$, two of which have a sagittal polarization while the third has a transverse polarization [13]. Nevertheless, for the proposed PnC strip, four native bands starts from $\omega=0$; those can be classified into bending mode with $x$-axis displacement $\left(\mathrm{B}_{\mathrm{x} 0}\right)$, bending mode with $y$-axis displacement $\left(\mathrm{B}_{\mathrm{y} 0}\right)$, bending mode with $z$-axis displacement $\left(\mathrm{B}_{z 0}\right)$, and torsional mode $\left(\mathrm{T}_{0}\right)$, based on the symmetry decomposition of displacements [25]. The other bands can be further categorized according to these four native modes. The curve of $T_{1}$ has discontinuous slopes when $k a / 2 \pi=0.125$. From the colour of the kinetic energetic ratios, we can know the band belongs to another mode when $k a / 2 \pi<0.125$, but not to $T_{1}$. For a given $k$, first ten eigen-frequencies are plotted. Hence these two bands have not been shown entirely in the figure. 
(a)

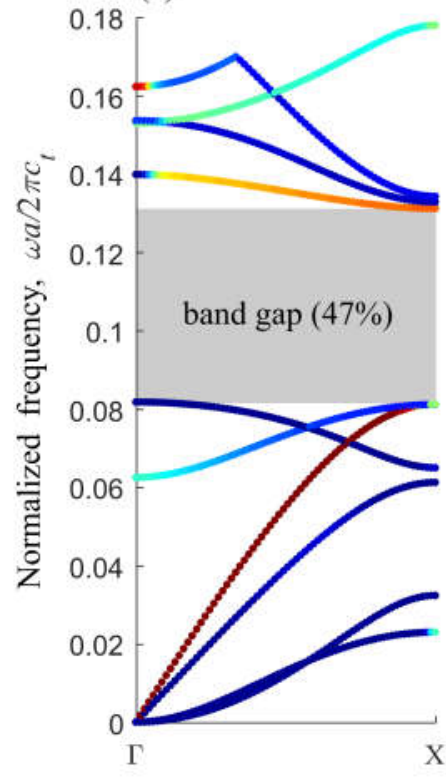

(b)

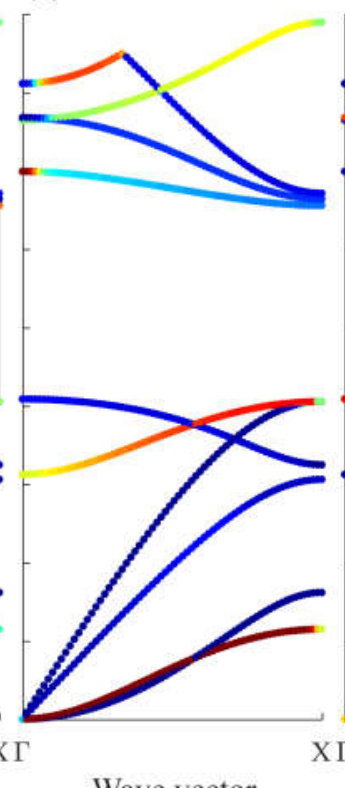

(c)

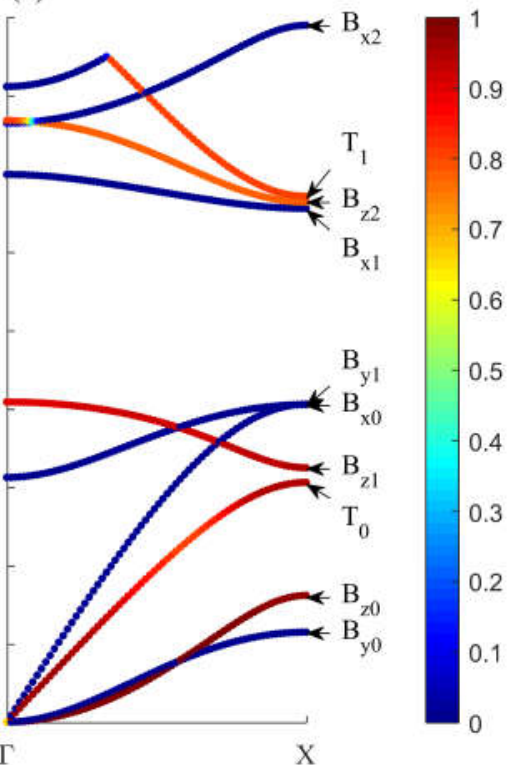

Figure 2. Band structure of the cross-like holey phononic crystal strip with representation of kinetic energy information, for parameters $b / a=0.625, c / a=0.25, d / a=0.325$ and $h / a=0.2$. The same band structure is plotted three times with colour representing kinetic energy contents of (a) the $x$, (b) the $y$, and (c) the $z$ polarizations of the elastic wave.

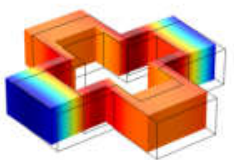

(a)

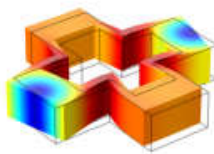

(f)

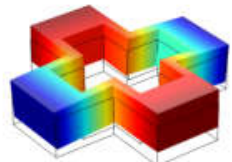

(b)

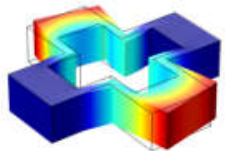

(g)

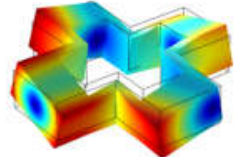

(c)

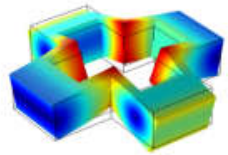

(h)

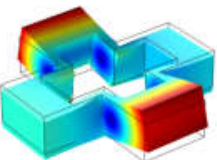

(d)

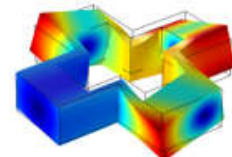

(i)

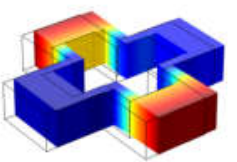

(e)

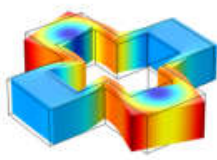

(j)

Figure 3. Modal shapes of the first ten frequency bands labeled in Figure 2 with normalized frequency $k a / 2 \pi=0.25$. (a) $\mathrm{B}_{\mathrm{y} 0}$, (b) $\mathrm{B}_{\mathrm{z} 0}$, (c) $\mathrm{T}_{0}$, (d) $\mathrm{B}_{\mathrm{z} 1}$, (e) $\mathrm{B}_{\mathrm{x} 0}$, (f) $\mathrm{B}_{\mathrm{y} 1}$, (g) $\mathrm{B}_{\mathrm{x} 1}$, (h) $\mathrm{B}_{\mathrm{z} 2}$, (i) $\mathrm{T}_{1}$, (j) $\mathrm{B}_{\mathrm{x} 2}$.

Figure 3 shows the modal shapes of the first ten frequency bands of figure 2, for normalized wavenumber $k a / 2 \pi=0.25$. Modal shapes are named in the figure according to their deformation shape. The modal shapes in figures 3(a) and 3(f) are for bending modes with $y$-axis displacement ( $\mathrm{B}_{\mathrm{y}}$-mode). Figures 3(b), 3(d) and 3(h) are for bending modes with $z$-axis displacement $\left(\mathrm{B}_{\mathrm{z}}\right.$-mode). Torsional modes (T-mode) appear in figures 3(c) and 3(i). Figures 3(e), 3(g) and 3(j) are for bending modes with $x$-axis displacement ( $\mathrm{B}_{\mathrm{x}}$-mode), they 
are longitudinal modes because they are mainly polarized in the propagation direction.

In figure 4 , the band structure of the cross-like holey phononic crystal strip is compared to two previously published models. Figure 4(a) presents the band structure of a "I"-like PnC strip cut from the square lattice holey PnC plate, with dimensions $r / a=0.36$ and $h / a=0.75$ [24]. The band structure of a similar strip cut from the hexagonal lattice holey plate is displayed in figure 4(b), with dimensions $a / d=3^{1 / 2}, r / d=0.45$, and $h / d=0.75$ [26]. Figure 4(c) is a copy of figure 1(a) shown for comparison. In order to eliminate the misinterpretation, the maximum values of vertical axis are written by a large bold font. The BG\% of main band gaps in figures 4(a)-4(c) are $10 \%, 48 \%$, and $47 \%$, respectively. Comparing with figure $4(\mathrm{a})$, figure $4(\mathrm{~b})$ has a wider and lower band gap. The only difference is that the first strip is cut from the square lattice while the second is cut from the hexagonal lattice. Hence, the model in figure 4(b) has one more geometry parameter than the one in figure 4(a). Comparing with the strip in figure 4(b), the proposed PnC strip in figure 4(c) has a much lower band gap while keeping about the same BG\%. The band gap of the proposed PnC strip has actually a much lower normalized center frequency than that of previously published PnC strips. Moreover, the thickness is only $h / a=$ 0.2 , while the strips in figures 4 (a) and 4(b) require the larger thicknesses $h / a=0.75$ and $h / d=$ $0.75(h / a=0.433)$, respectively. In addition, Pennec et al. discussed a strip whose unit cell contains a circular hole in the middle and two symmetric stubs on the sides. Thickness $h / a=$ 0.44 was applied to induce a maximum $\mathrm{BG} \%$ of $14.8 \%$ [38].

An example is given to show intuitively. Three kinds of strips in figure 4 are designed to meet the working frequency from $7.24 \mathrm{MHz}$ to $8.11 \mathrm{MHz}$ of the acoustic microwaveguide [39]. For the strip in figure 4(a), $a=385 \mu \mathrm{m}$, the band gap appears from $7.28 \mathrm{MHz}$ to 8.06 MHz. So the band gap is not broad enough to meet the requirement; For the strip in figure 4(b), $a=200 \mu \mathrm{m}$, the band gap locates from $6.77 \mathrm{MHz}$ to $11.09 \mathrm{MHz}$; For the proposed strip in figure 4(c), $a=60 \mu \mathrm{m}$, the band gap spans from $6.40 \mathrm{MHz}$ to $10.30 \mathrm{MHz}$. Therefore, as for the structural size, the proposed strip is smaller than one-third of the strip in figure 4(b). On the other hand, with a same lattice constant $(a=200 \mu \mathrm{m})$, the proposed strip can induce a much lower frequency band gap from $1.92 \mathrm{MHz}$ to $3.09 \mathrm{MHz}$ than the strip in figure 4(b). Consequently, the proposed PnC strip has at least three advantages over previously reported PnC strips: a broader or equivalent band gap, a lower frequency band gap, and a smaller size. 


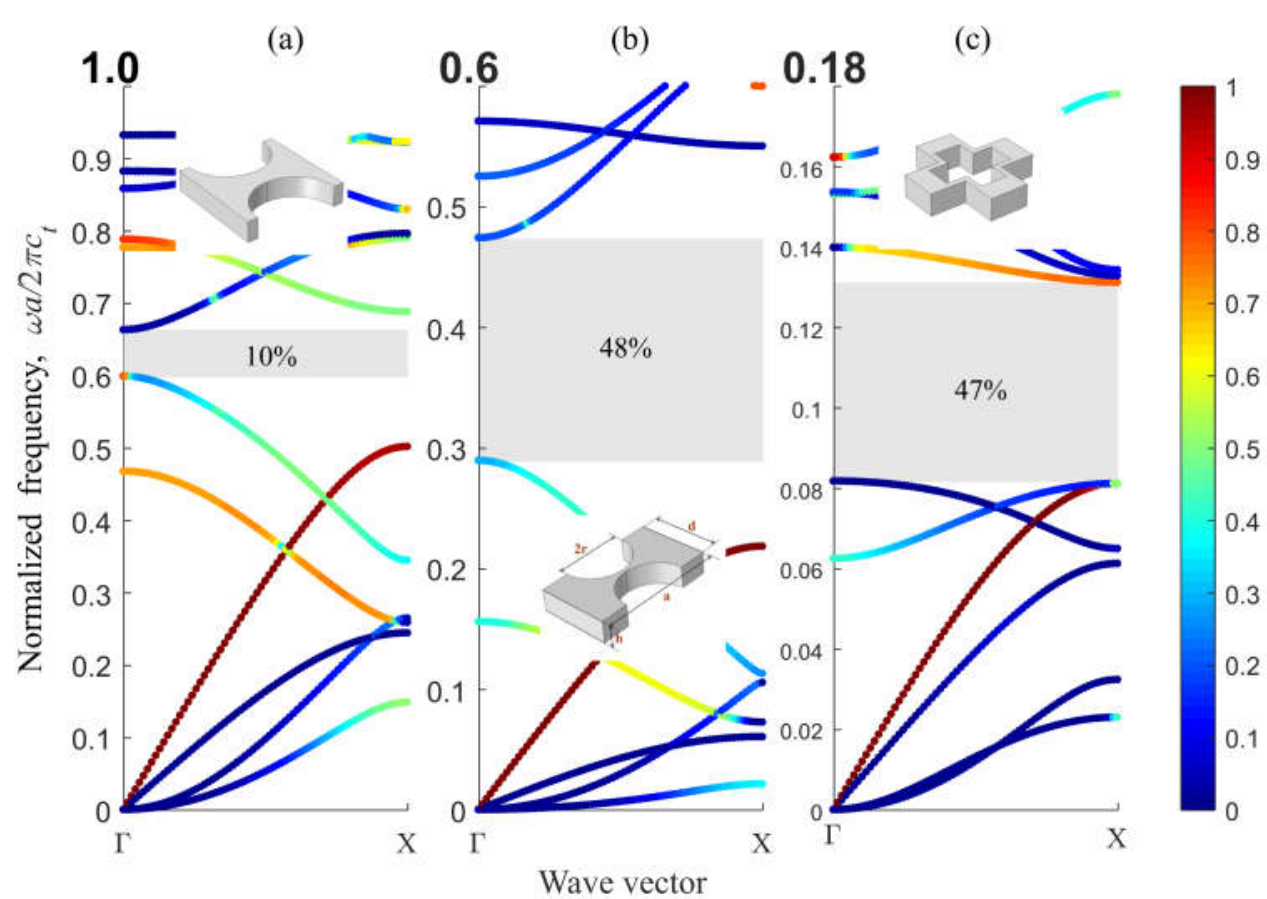

Figure 4. Band structures of (a) the PnC strip cut from the square-lattice holey PnC plate $(r / a=$ $0.36, h / a=0.75)$, (b) the PnC strip cut from hexagonal-lattice holey PnC plate $\left(a / d=3^{1 / 2}, r / d=\right.$ 0.45 and $h / d=0.75)$, and (c) the proposed PnC strip $(b / a=0.625, c / a=0.25, d / a=0.325$ and $h / a=0.2$ ). Colour bars represent the kinetic energy content in the $x$-axis polarization of the elastic wave.

To obtain the largest value of $\mathrm{BG} \%$, the optimization of geometry parameters was conducted inductively. Geometry parameters can be divided into two groups as in-plane parameters and out-of-plane parameter. The in-plane geometry parameters include dimensions of holes $b, c$ and $d$. The thickness $h$ is the out-of-plane geometry parameter. The in-plane parameters have an important influence on all modes at the same time. However, the out-of-plane parameter only has a significant impact on out-of-plane modes, $\mathrm{B}_{\mathrm{z}}$-modes and T-modes. Therefore, we consider the effect of the geometry parameters on the band structure, from the dimensions of the cross-like hole $b$ and $c$, to the length of square holes $d$, and finally to the thickness $h$ of the strip.

Figure 5 illustrates the effect of geometry parameters of the cross-like hole on the band gap. The variation of the band gap is treated as a function of $b / a$ and $c / a$, where $w / a=0.05$ and $h / a=0.2$ are fixed. Parameter $d$ changes with $c$ due to $c+d+2 w=a$. As shown in figure 5, band gaps with a large $\mathrm{BG} \%(>40 \%)$ are available within a large region of geometry parameters $b$ and $c$. The maximum $47 \%$ of BG\% locates at the optimal geometry parameters $(b / a, c / a)$ equal $(0.625,0.25)$. For a given $c / a$, the $\mathrm{BG} \%$ reaches a maximum at an intermediate 
value of $b / a$ and decreases as $b / a$ deviates from this value. This is quite different from previously reported strips for which the maximum of BG\% appears only as the geometry size closes to its limit (i.e. $r / a \sim 0.5$ ). For the proposed strip, an intermediate $b / a$ means an intermediate filling fraction of solid material since the filling fraction of solid material decreases with increase of $b / a$ when the other geometry parameters are given. Hence, intermediate filling fractions can lead to the maximums of $\mathrm{BG} \%$. As a note, intermediate filling fractions escape the shortcomings that come with small filling fractions, such as mechanical instability and low bearing capacity. Furthermore, they also escape the disadvantages that come with large filling fractions, such as overweight.

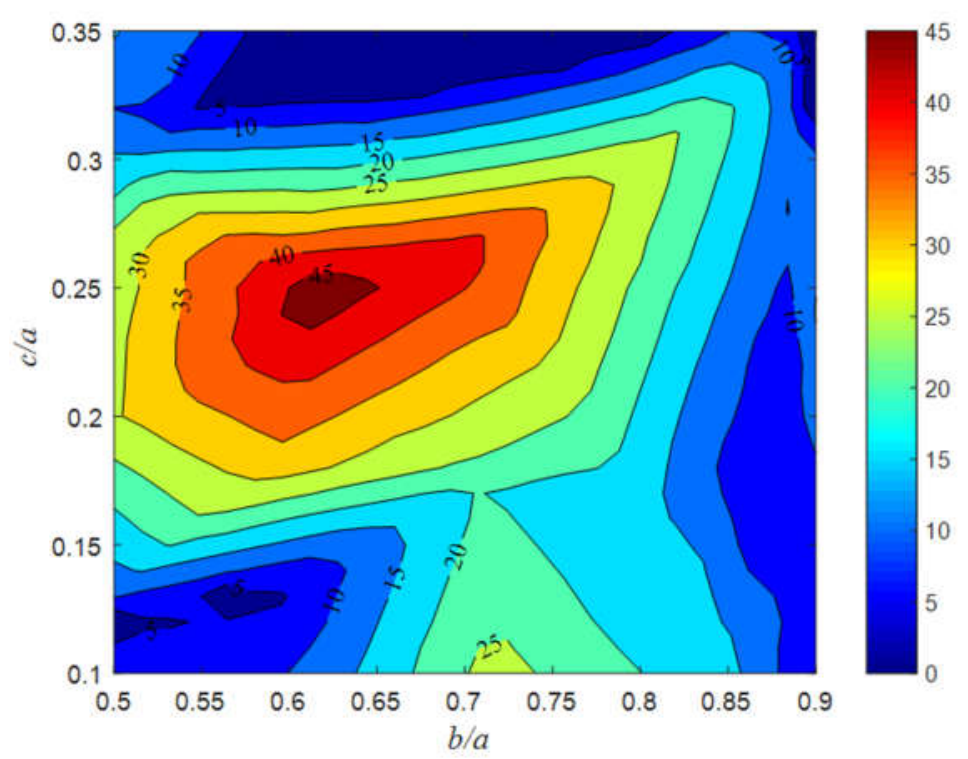

Figure 5. BG\% is illustrated by colour as a function of geometry parameters $b / a$ and $c / a$, with $w / a=0.05$ and $h / a=0.2$. The maximum $47 \%$ of BG\% locates $(0.625,0.25)$. 


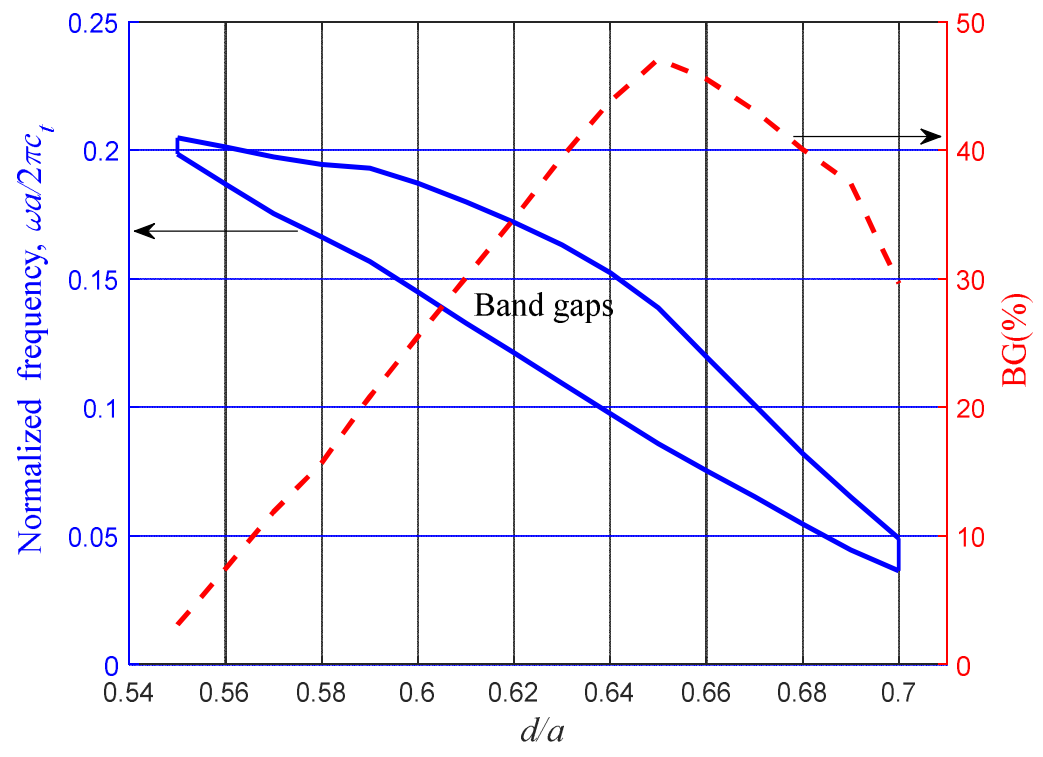

Figure 6. Variation of center frequency and band gap width as a function of $d / a$, where $b / a=$ $0.625, c / a=0.25$, and $h / a=0.2$. The maximum value of BG\% appears for $d / a=0.65$.

Figure 6 illustrates the variation of the band gap versus the normalized length $d / a$ of the square holes at the corners. The other parameters are fixed at $b / a=0.625, c / a=0.25$, and $h / a=$ 0.2. As $d / a$ increases, the lower and the upper boundaries of the band gap both decrease. Actually, an increase in $d / a$ decreases the bending stiffness along the three axes $(x, y$ and $z)$ and also decreases the torsion stiffness. Consequently, all bending and torsion bands move down to lower frequencies. Nevertheless, different bands have different degrees of descent. Thus the lower and upper boundaries of the band gap are sequentially limited by bands of different symmetries. Furthermore, as the red dash line reveals, there exists an optimum $d / a$ of 0.325 with respect to the maximum $\mathrm{BG} \%$, further confirming that an intermediate filling fraction can induce the maximum $\mathrm{BG} \%$ for the proposed strip. 


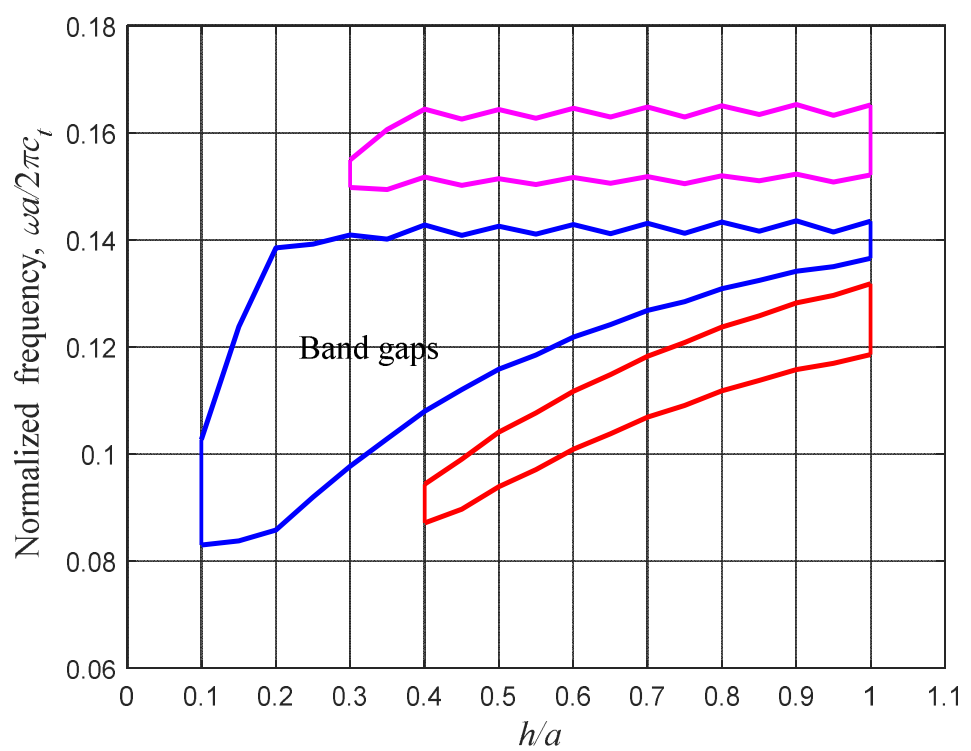

Figure 7. Variation of the band gaps as a function of the parameter $h / a$, where $b / a=0.625, c / a$ $=0.25$ and $d / a=0.65$ are fixed. The main band gap reaches maximum width at $h / a=0.2$.

Figure 7 illustrates the effect of the thickness $h$ on the band structure of the PnC strip. The in-plane geometry parameters are fixed at $b / a=0.625, c / a=0.25$, and $d / a=0.325$. With the increase of $h / a$, two band gaps are formed at first, and then the third band gap appears when $h / a>0.3$. The colours are used to distinguish different band gaps. As noted above, the thickness $h$ just affects modes related to $z$-axis, such as $\mathrm{B}_{\mathrm{z}}$-mode and T-mode. The out-of-plane bending stiffness and torsion stiffness both increase with the increase in parameter $h$, but the in-plane bending stiffness is independent of $h$. As a result, the bands of the $\mathrm{B}_{\mathrm{z}}$-mode and the T-mode both change with the variation of $h / a$, while the bands of the $\mathrm{B}_{\mathrm{x}}$-mode and the $\mathrm{B}_{\mathrm{y}}$-mode remain stationary. Specifically, with an increase of $h / a$, the bands of the $\mathrm{B}_{z 0}$-mode and the $\mathrm{T}_{0}$-mode become steeper, while the bands of higher-order bending and torsion modes move up entirely. When the bands of the $\mathrm{B}_{\mathrm{z}}$-mode and the T-mode move inside, the main band gap previously determined by the $\mathrm{B}_{\mathrm{x}}$-mode and the $\mathrm{B}_{\mathrm{y}}$-mode becomes separated into two or three parts. From this point of view, the best strategy to obtain the widest band gap is to prevent the bands of the $\mathrm{B}_{\mathrm{z}}$-mode and the T-mode from entering the band gap determined by the $\mathrm{B}_{\mathrm{x}}$-mode and the $\mathrm{B}_{\mathrm{y}}$-mode. As shown in figure 7 , the first band gap reaches its maximum width at $h / a=0.2$. When $h / a>0.3$, the main band gap is separated by the bands of the $\mathrm{B}_{\mathrm{z}}$-mode and the T-mode, and more gaps appear.

Next, we investigate the dependence of the band gaps on the width of narrow connectors 
$w / a$. In the above discussion, the width of the narrow connectors $w / a$ was fixed as 0.05 . This parameter is worth much attention since the width of connectors has a significant effect on the lumps-connectors system. The maximums of $\mathrm{BG} \%$ for different widths $w / a$ and the corresponding optimal geometry parameters are listed in Table 1. Both the upper and the lower edges of band gaps decrease with a decrease in $w / a$. However, there is an optimal value $w / a$ of 0.05 which makes the band gap reach the maximum of $\mathrm{BG} \%$. Compared to a slim connector with a small $w / a$, a relatively thick connector means robustness of the PnC strip. For the corresponding geometry parameters, $c / a$ increases monotonically with decreasing $w / a$, and the optimal $h / a$ is always around 0.2 .

Table 1. The maximums of BG\% for different connector widths $w / a$ and the corresponding optimal geometry parameters $b / a, c / a, d / a$ and $h / a$.

\begin{tabular}{cccccc}
\hline$w / a$ & $f_{\text {top }}$ & $f_{\text {bot }}$ & $\mathrm{BG} \%$ & $(b / a, c / a, d / a)$ & $h / a$ \\
\hline 0.070 & 0.113 & 0.174 & $42.62 \%$ & $(0.625,0.225,0.635)$ & 0.20 \\
0.065 & 0.108 & 0.168 & $43.50 \%$ & $(0.625,0.225,0.645)$ & 0.21 \\
0.060 & 0.096 & 0.151 & $44.21 \%$ & $(0.625,0.225,0.655)$ & 0.20 \\
0.055 & 0.088 & 0.140 & $45.33 \%$ & $(0.675,0.250,0.640)$ & 0.20 \\
0.050 & 0.082 & 0.132 & $46.73 \%$ & $(0.625,0.250,0.650)$ & 0.20 \\
0.045 & 0.079 & 0.126 & $45.40 \%$ & $(0.600,0.250,0.660)$ & 0.22 \\
0.040 & 0.071 & 0.112 & $44.59 \%$ & $(0.625,0.275,0.645)$ & 0.20 \\
0.035 & 0.064 & 0.100 & $44.39 \%$ & $(0.600,0.275,0.655)$ & 0.20 \\
0.030 & 0.048 & 0.073 & $41.79 \%$ & $(0.625,0.275,0.665)$ & 0.20 \\
0.025 & 0.045 & 0.069 & $42.02 \%$ & $(0.600,0.300,0.650)$ & 0.20 \\
\hline
\end{tabular}

According to the Bragg scattering mechanism, the spatial modulation must be commensurate with the wavelength in the band gap. Unlike conventional convex holey PnC strips, the proposed cross-like holey strip has a folding topology. This folding-type topology extends the flexural wave propagation path but the structure size remains small. Alternatively, the unit cell can also be regarded as four lumps connected by four narrow connectors. From the above discussion, the widest band gap is determined by the $\mathrm{B}_{\mathrm{x}}$-mode and the $\mathrm{B}_{\mathrm{y}}$-mode. Observing those modes in figure 3, it is seen that the lumps vibrate like rigid bodies since their bending stiffness is much larger than that of the connectors. The low-frequency flexural wave is thus effectively modulated to open a band gap by the lump-connector system, owing to the large difference between their bending stiffness. 


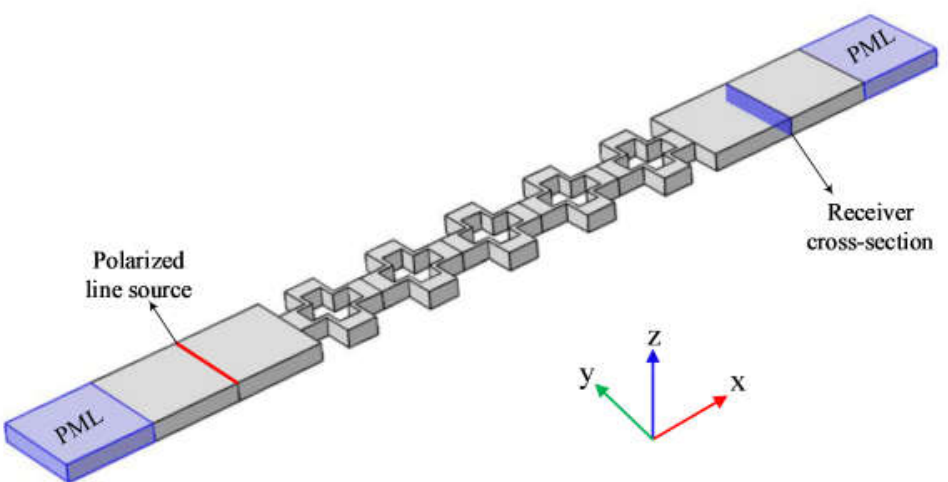

Figure 8. Schematic setting used for the computation of transmission through five periods of the cross-like holey phononic crystal strip.

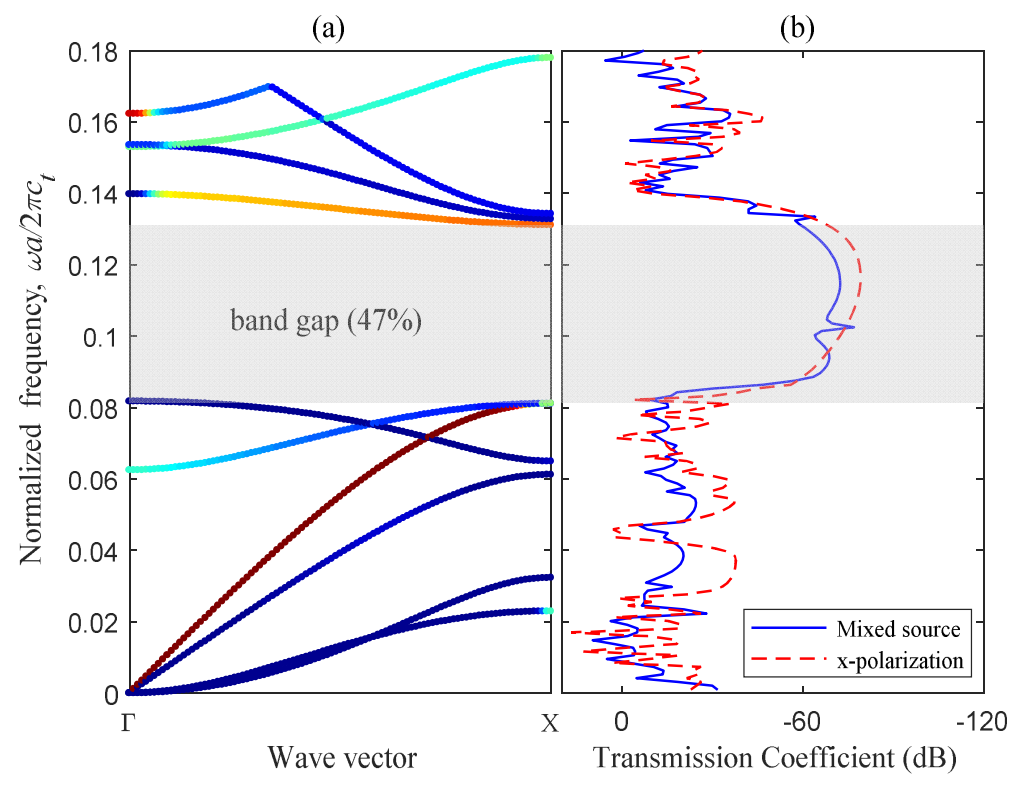

Figure 9. Band structure and transmission spectra for elastic wave in the PnC strip with parameters $b / a=0.625, c / a=0.25, d / a=0.325$, and $h / a=0.2$. (a) Band structure for PnC strip. Colour bar represents kinetic energy content in the $x$-axis polarization of the elastic wave. (b) Transmission spectra of elastic waves through a finite strip structure with five periods, for two different wave sources.

Furthermore, to evaluate performance of the proposed PnC strip on vibration isolation, the transmission spectrum is calculated to characterize the excitation response of the PnC strip. The transmission spectrum is often used to evaluate wave propagation properties for a finite periodic structure. In addition, frequency ranges with large attenuation in the transmission spectrum for the finite periodic structure can be directly compared to the band gaps in the band structure for the infinite periodic system.

The analyzed configuration is depicted as figure 8 . The polarized line source can be selected to have either $x$-, $y$-, $z$-, or mixed-polarization. Perfectly match layers (PML) are 
added at the two ends of the structure to avoid reflections of outgoing waves [40]. Figure 9(b) plots the calculated transmission spectra for a finite PnC strip with five periods. A normalized frequency range with strong attenuation from 0.082 to 0.141 can be clearly seen from the transmission spectra for a mixed-polarized source and an $x$-polarized source. This frequency range matches fairly well with the band gap from 0.082 to 0.132 predicted by the band structure in figure 9(a). The small difference in the upper edge frequencies might be attributed to the fact that some modes have not been excited by the polarized line sources, i.e. they are deaf.

\section{Conclusion}

A cross-like holey PnC strip has been proposed with cross-like holes in the center and square holes at the corners in a square lattice. The band structure, the eigenmodes and the transmission characteristics of the PnC strip have been analyzed by using FEM. The maximum of the relative band gap width reaches $47 \%$ after design. Compared with previously reported topologies with the same lattice constant, the proposed strip induces much lower frequency band gaps. At the same time, a much smaller thickness is required. Combining the two advantages of low-frequency band gap and small size/weight, the proposed PnC strip has a potential of application in the field of MEMS. Its intermediate filling fraction overcomes the shortcomings of phononic crystal strips with either small or large filling fractions. The proposed PnC strip could be applied as a vibration isolator or a noise insulator.

\section{Acknowledgment}

This work was supported by the National Natural Science Foundation of China (11272126, 51435006 and 51421062), the Fundamental Research Funds for the Central Universities, HUST: 2016JCTD114 and 2015TS121. Hu and Jiang also acknowledge the support from China Scholarship Council (Grants Nos. 201606165026 and 201606160045).

\section{References}

[1] Khelif A and Adibi A 2015 Phononic Crystals: Fundamentals and Applications (New York, Springer)

[2] Laude V 2015 Phononic Crystals: Artificial Crystals for Sonic, Acoustic, and Elastic Waves (Berlin/Boston, Walter de Gruyter GmbH)

[3] Safavi-Naeini A H and Painter O 2010 Design of optomechanical cavities and waveguides on a 
simultaneous bandgap phononic-photonic crystal slab Opt. Expres. 18 14926-43

[4] Safavi-Naeini A H, Hill J T, Meenehan S, Chan J, Groblacher S and Painter O 2014

Two-dimensional phononic-photonic band gap optomechanical crystal cavity Phys. Rev. Lett. 112 153603

[5] Wang Y F, Wang Y S and Su X X 2011 Large bandgaps of two-dimensional phononic crystals with cross-like holes J. Appl. Phys. 110113520

[6] Mousanezhad D, Babaee S, Ghosh R, Mahdi E, Bertoldi K and Vaziri A 2015 Honeycomb phononic crystals with self-similar hierarchy Phys. Rev. B 92

[7] Jiang S, Dai L X, Chen H, Hu H P, Jiang W and Chen X D 2017 Folding beam-type piezoelectric phononic crystal with low-frequency and broad band gap Appl. Math. Mech-Engl. 38 411-22

[8] Jiang S, Chen H, Dai L X, Hu H P and Laude V 2017 Multiple low-frequency broad band gaps generated by a phononic crystal of periodic circular cavity sandwich plates Compos. Struct. 176 294-303

[9] Dai L X, Jiang S, Lian Z Y, Hu H P and Chen X D 2015 Locally resonant band gaps achieved by equal frequency shunting circuits of piezoelectric rings in a periodic circular plate $J$. Sound. Vib. 337 $150-60$

[10] Song Y B, Feng L P, Wen J H, Yu D L and Wen X S 2015 Reduction of the sound transmission of a periodic sandwich plate using the stop band concept Compos. Struct. 128 428-36

[11] Zhang H, Wen J H, Xiao Y, Wang G and Wen X S 2015 Sound transmission loss of metamaterial thin plates with periodic subwavelength arrays of shunted piezoelectric patches J. Sound. Vib. 343 104-20

[12] Wang P, Casadei F, Shan S C, Weaver J C and Bertoldi K 2014 Harnessing buckling to design tunable locally resonant acoustic metamaterials Phys. Rev. Lett. 113014301

[13] Khelif A, Aoubiza B, Mohammadi S, Adibi A and Laude V 2006 Complete band gaps in two-dimensional phononic crystal slabs Phys. Rev. E 74046610

[14] Coffy E, Lavergne T, Addouche M, Euphrasie S, Vairac P and Khelif A 2015 Ultra-wide acoustic band gaps in pillar-based phononic crystal strips J Appl Phys 118214902

[15] Lian Z Y, Hu H P, Dai L X, Luo B, Liang Y X and Chen X D 2017 Coupling between two kinds of band gaps of a shunted tube piezoelectric phononic crystal J Intell Mater Syst Struct 28 2153-66

[16] Dong H W, Wang Y S and Zhang C Z 2017 Topology Optimization of Chiral Phoxonic Crystals With Simultaneously Large Phononic and Photonic Bandgaps IEEE Photon. J. 9 1-16

[17] Hedayatrasa S, Abhary K, Uddin M and Ng C T 2016 Optimum design of phononic crystal perforated plate structures for widest bandgap of fundamental guided wave modes and maximized in-plane stiffness J. Mech. Phys. Solids 89 31-58

[18] Hussein M I, Leamy M J and Ruzzene M 2014 Dynamics of Phononic Materials and Structures: Historical Origins, Recent Progress, and Future Outlook Appl. Mech. Rev. 66040802

[19] Cummer S A, Christensen J and Alù A 2016 Controlling sound with acoustic metamaterials Nat. Rev. Mater. 116001

[20] Wu T T, Wang W S, Sun J H, Hsu J C and Chen Y Y 2009 Utilization of phononic-crystal reflective gratings in a layered surface acoustic wave device Appl. Phys. Lett. 94101913

[21] Huang C Y, Sun J H and Wu T T 2010 A two-port ZnO/silicon Lamb wave resonator using phononic crystals Appl. Phys. Lett. 97031913

[22] Wang N, Tsai J M-L, Hsiao F L, Soon B W, Kwong D L, Palaniapan M and Lee C K 2012 Micromechanical resonators based on silicon two-dimensional phononic crystals of square lattice IEEE $J$. Microelectromech. Syst. 21 801-10 
[23] Hsu J C, Wu T T and Hsu H S 2013 Measurement of frequency gaps and waveguiding in phononic plates with periodic stepped cylinders using pulsed laser generated ultrasound J. Appl. Phys. 113083511 [24] Hsu F C, Lee C I, Hsu J C, Huang T C, Wang C H and Chang P 2010 Acoustic band gaps in phononic crystal strip waveguides Appl. Phys. Lett. 96051902

[25] Hsu F C, Hsu J C, Huang T C, Wang C H and Chang P 2011 Reducing support loss in micromechanical ring resonators using phononic band-gap structures J. Phys. D: Appl. Phys. 44375101

[26] Feng D, Xu D H, Wu G Q, Xiong B and Wang Y L 2013 Extending of band gaps in silicon based one-dimensional phononic crystal strips Appl. Phys. Lett. 103151906

[27] Feng D, Xu D H, Wu G Q, Xiong B and Wang Y L 2014 Phononic crystal strip based anchors for reducing anchor loss of micromechanical resonators J. Appl. Phys. 115024503

[28] Bahr B, Marathe R and Weinstein D 2015 Theory and design of phononic crystals for unreleased CMOS-MEMS resonant body transistors IEEE J. Microelectromech. Syst. 24 1520-33

[29] Mohammadi S, Eftekhar A A, Hunt W D and Adibi A 2009 High-Q micromechanical resonators in a two-dimensional phononic crystal slab Appl. Phys. Lett. 94051906

[30] Soliman Y M, Su M F, Leseman Z C, Reinke C M, El-Kady I and Olsson III R H 2010 Phononic crystals operating in the gigahertz range with extremely wide band gaps Appl. Phys. Lett. 97193502

[31] Li C S, Huang D, Guo J R and Nie J J 2013 Engineering of band gap and cavity mode in phononic crystal strip waveguides Phys. Rev. A 377 2633-7

[32] Wu T C, Wu T T and Hsu J C 2009 Waveguiding and frequency selection of Lamb waves in a plate with a periodic stubbed surface Phys. Rev. B 79104306

[33] Bilal O R and Hussein M I 2013 Trampoline metamaterial: Local resonance enhancement by springboards Appl Phys Lett 103111901

[34] Ma C Y, Guo J W and Liu Y F 2015 Extending and lowing band gaps in one-dimensional phononic crystal strip with pillars and holes J. Phys. Chem. Solids 87 95-103

[35] Shu F F, Liu Y S, Wu J F and Wu Y H 2016 Band gap in tubular pillar phononic crystal plate Ultrasonics 71 172-6

[36] Jin Y B, Pennec Y, Pan Y D and Djafari-Rouhani B 2016 Phononic crystal plate with hollow pillars connected by thin bars J. Phys. D: Appl. Phys. 50035301

[37] Kim T and Seo C 2000 A novel photonic bandgap structure for low-pass filter of wide stopband IEEE Microw. Guided. Wave. Lett. 10 13-5

[38] Pennec Y, Djafari-Rouhani B, Li C, Escalante J, Martínez A, Benchabane S, Laude V and Papanikolaou N 2011 Band gaps and cavity modes in dual phononic and photonic strip waveguides AIP $A d v 1041901$

[39] Feng D, Jiang W, Xu D, Xiong B and Wang Y 2017 Micro-silicon phononic crystal with locally resonant theory Appl Phys Lett 110 2022-583

[40] Diatta A, Kadic M, Wegener M and Guenneau S 2016 Scattering problems in elastodynamics Phys. Rev. B 94100105 